\title{
Dynamic Imine Bond-Based Shape Memory Polymers with Permanent Shape Reconfigurability for 4D Printing
}

Jia-Tao Miao, ${ }^{\dagger}$ Meiying Ge,$^{\dagger}$ Shuqiang Peng, ${ }^{\dagger}$, Jie Zhong, ${ }^{\dagger}$ Yuewei Li, ${ }^{\dagger}$, Zixiang Weng, ${ }^{\dagger}$ Lixin $W u, *^{\dagger}$ and Longhui Zheng* ${ }^{\prime}, *$

$\uparrow$ CAS Key Laboratory of Design and Assembly of Functional Nanostructures, Fujian Key Laboratory of Nanomaterials, Fujian Institute of Research on the Structure of Matter, Chinese Academy of Sciences, Fuzhou 350002, People's Republic of China.

* Fujian Universities and Colleges Engineering Research Center of Soft Plastic Packaging Technology for Food, Fuzhou 350300, People's Republic of China

${ }^{\S}$ University of Chinese Academy of Sciences, Beijing 100049, People’s Republic of China

Corresponding Authors

*E-mail address: lxwu@fjirsm.ac.cn (LX. Wu), lhzheng@ fjirsm.ac.cn (LH. Zheng) 


\section{Index}

Figure S1. Dynamic reversible exchange mechanism of imine bonds.................................S-3

Figure S2. Synthesis routes of MEFB (a) and HPASi (b) .................................................

Figure S3. ${ }^{1} \mathrm{H}$ NMR (top) and ${ }^{13} \mathrm{C}$ NMR (bottom) spectra of MEFB.................................. S-4

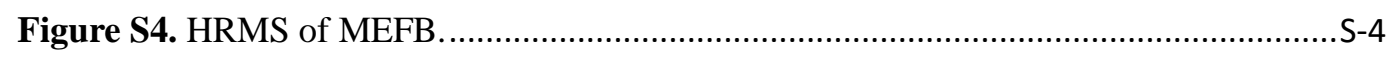

Figure S5. ${ }^{1} \mathrm{H}$ NMR spectra of 3-aminopropyltriethoxysilane (top) and HPASi (bottom)....S-5

Figure S6. FTIR spectra of IEM (a), IEMSi1 (b), IEMSi2 (c) and IEMSi3 (d), ................... S-5

Figure S7. $E^{\prime}\left(T_{\mathrm{fix}}\right) / E^{\prime}\left(T_{\mathrm{prog}}\right)$ ratio of IEMSis.................................................................

Figure S8. DMA thermograms for storage modulus (a) and $\tan \delta$ (b) against temperature of

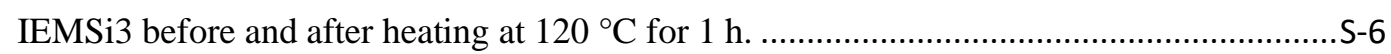

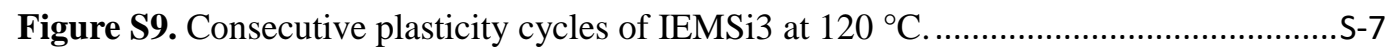

Figure S10. Digital photo of IEMSi3 reconfigured for three times and can still bear a weight of $200 \mathrm{~g}$ S-7 

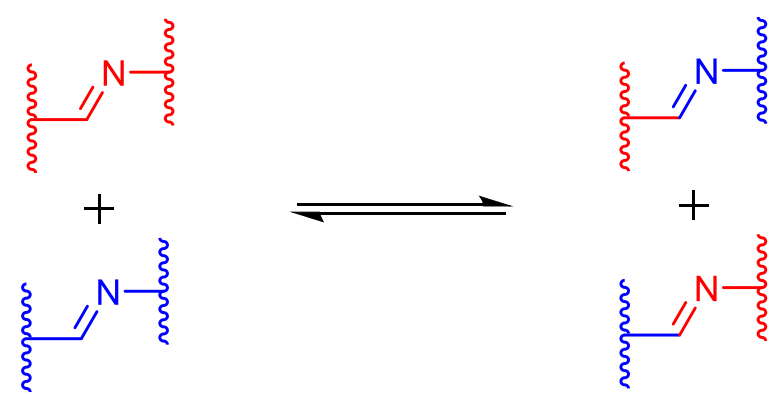

Figure S1. Dynamic reversible exchange mechanism of imine bonds.

(a)

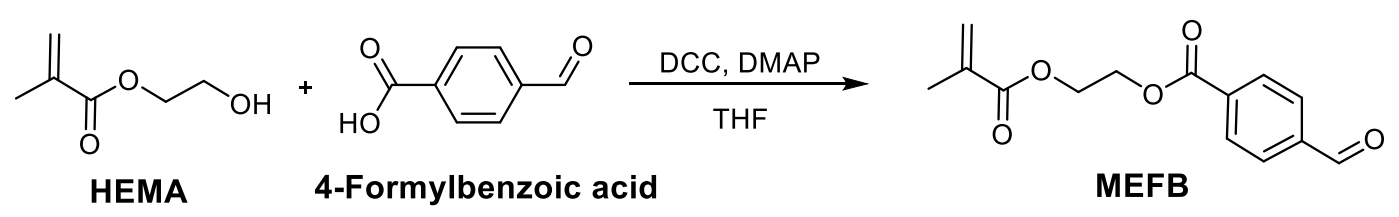

(b)
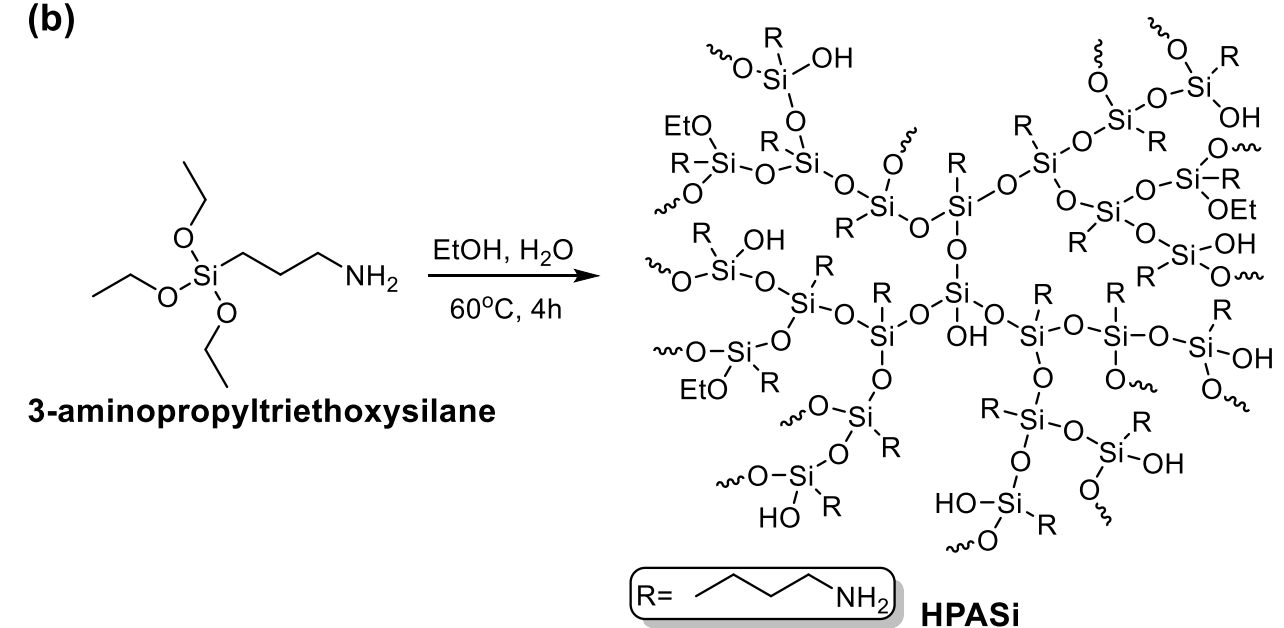

Figure S2. Synthesis routes of MEFB (a) and HPASi (b). 


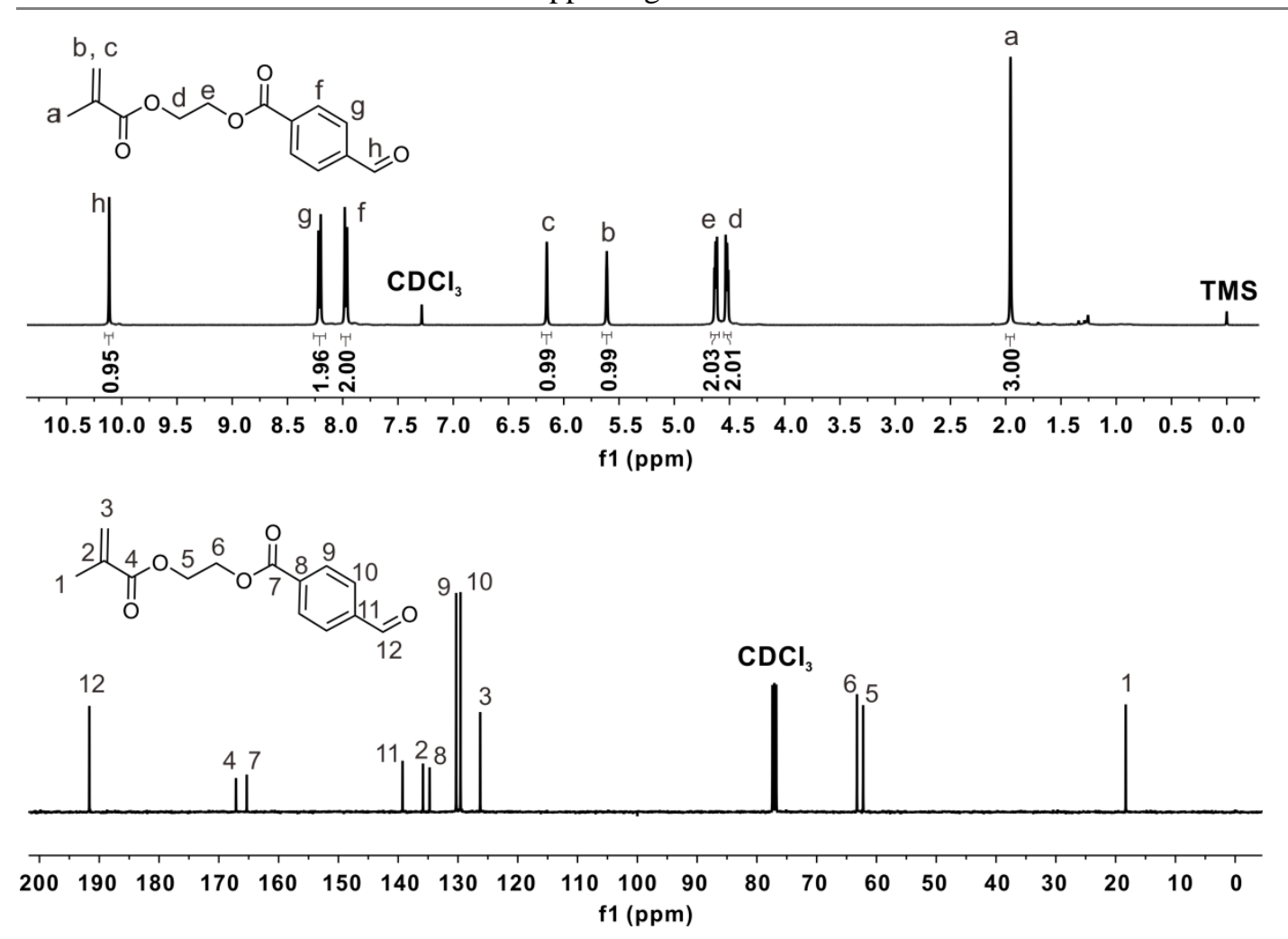

Figure S3. ${ }^{1} \mathrm{H}$ NMR (top) and ${ }^{13} \mathrm{C}$ NMR (bottom) spectra of MEFB.

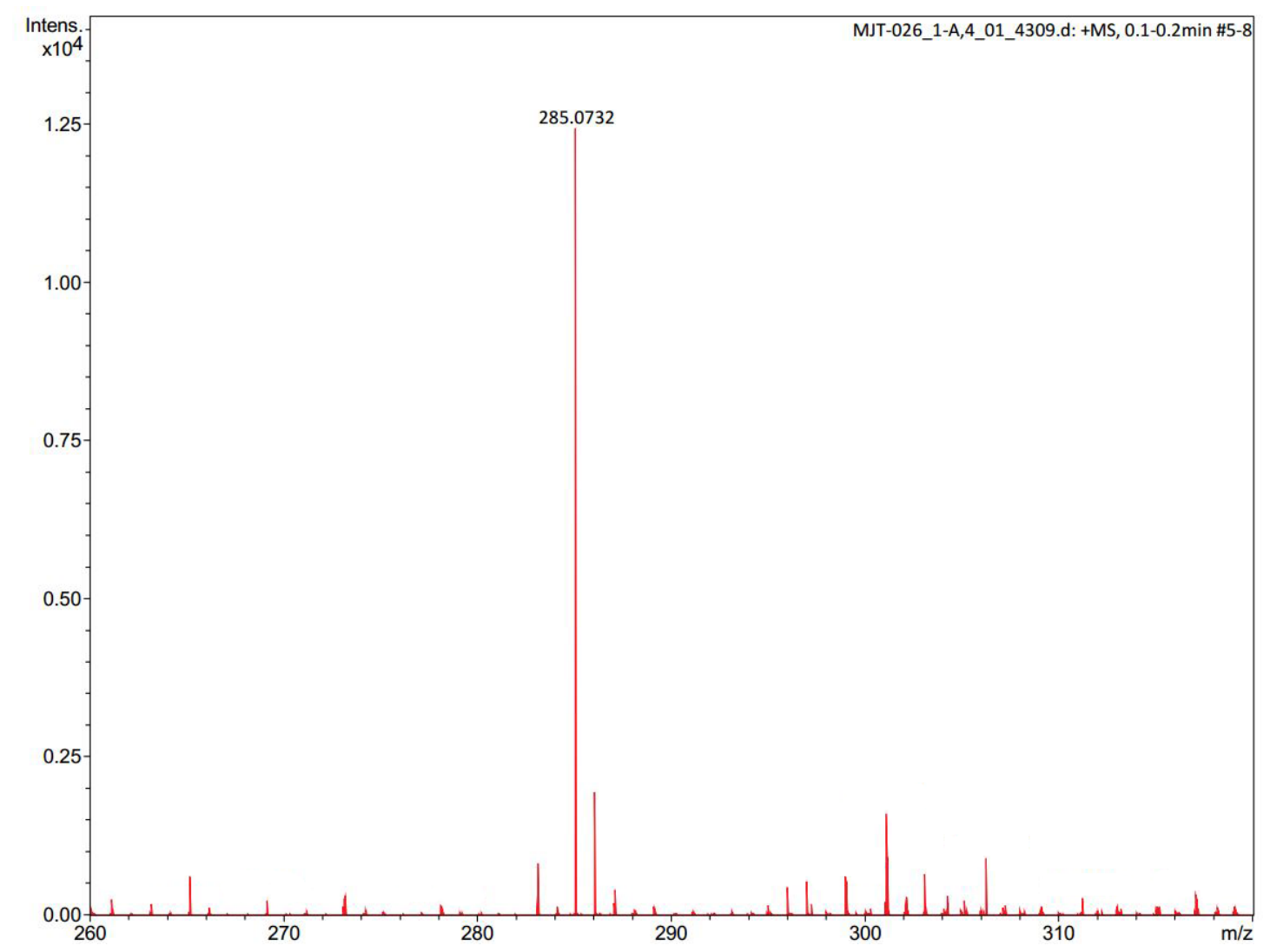

Figure S4. HRMS of MEFB. 


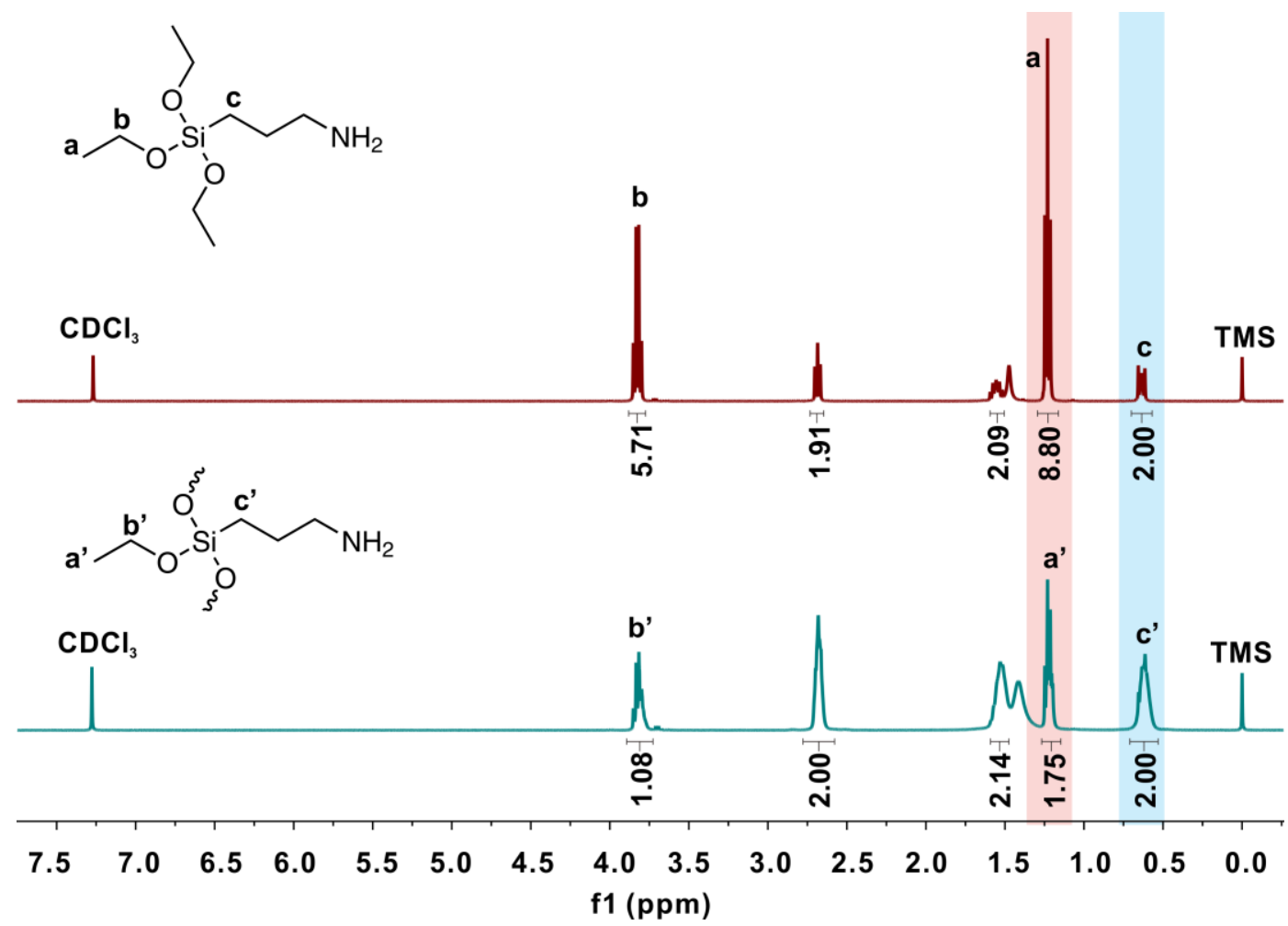

Figure S5. ${ }^{1} \mathrm{H}$ NMR spectra of 3-aminopropyltriethoxysilane (top) and HPASi (bottom).

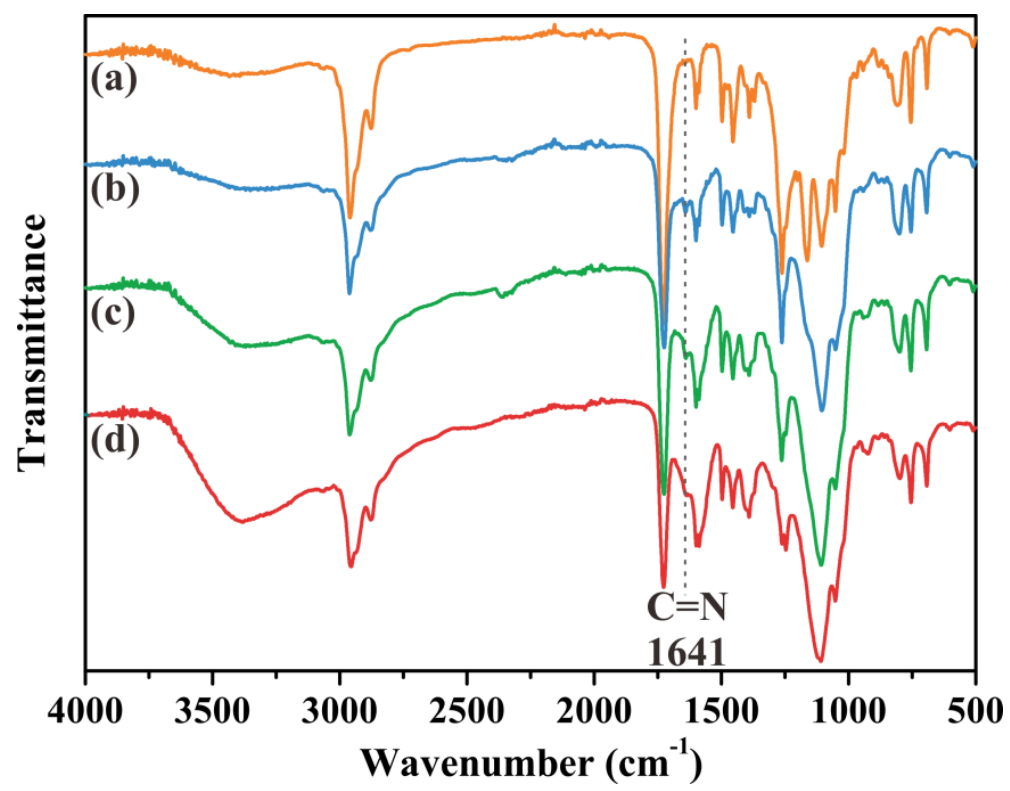

Figure S6. FTIR spectra of IEM (a), IEMSi1 (b), IEMSi2 (c) and IEMSi3 (d). 


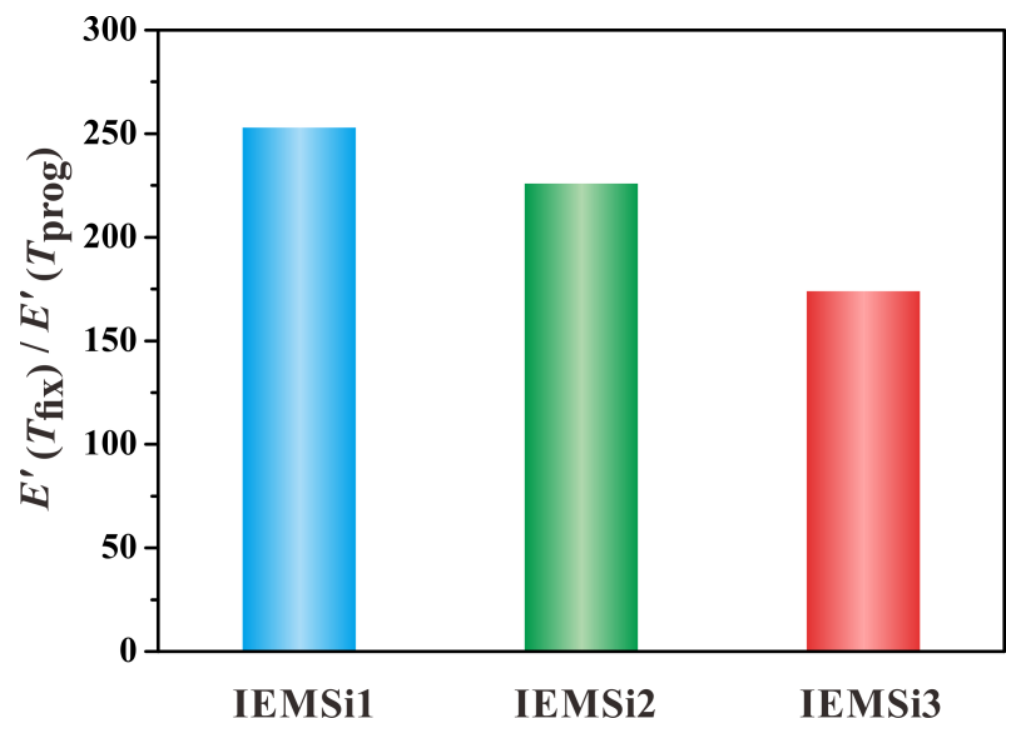

Figure S7. $E^{\prime}\left(T_{\mathrm{fix}}\right) / E^{\prime}\left(T_{\text {prog }}\right)$ ratio of IEMSis.
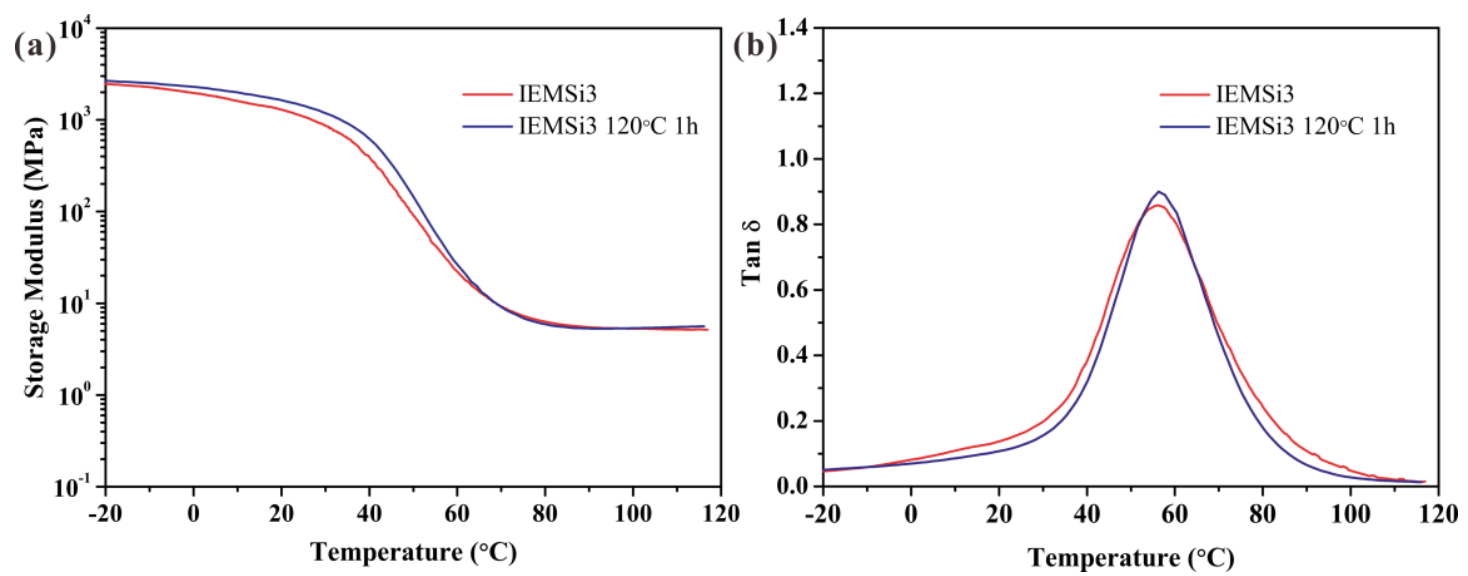

Figure S8. DMA thermograms for storage modulus (a) and $\tan \delta$ (b) against temperature of IEMSi3 before and after heating at $120^{\circ} \mathrm{C}$ for $1 \mathrm{~h}$. 


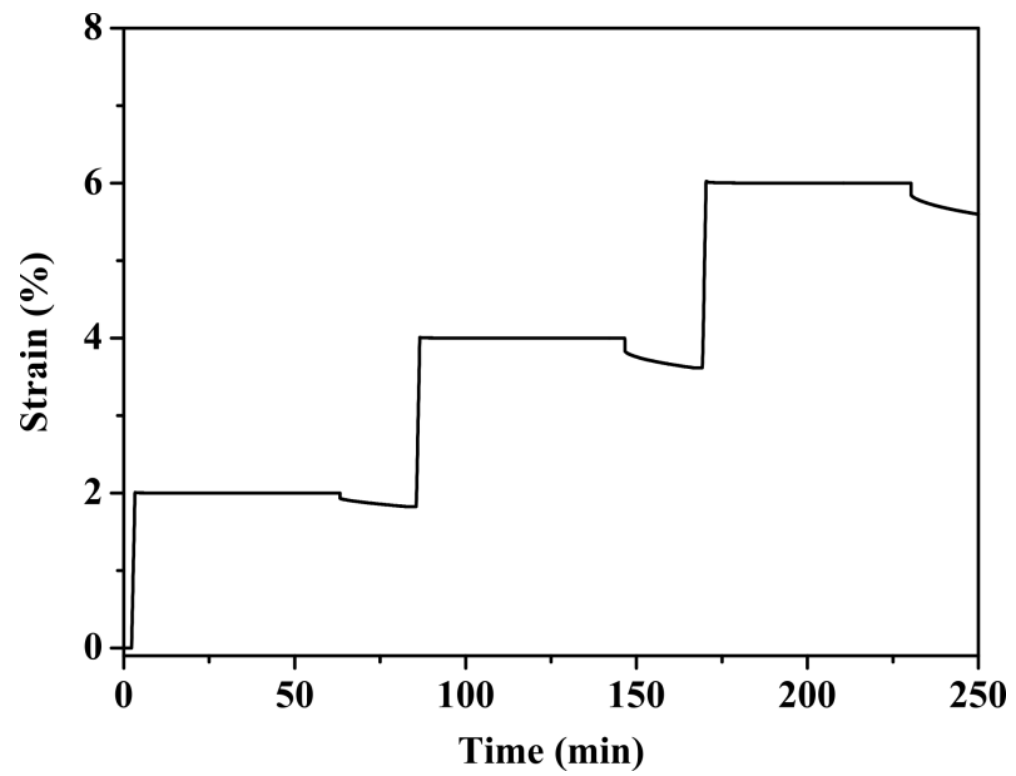

Figure S9. Consecutive plasticity cycles of IEMSi3 at $120^{\circ} \mathrm{C}$.

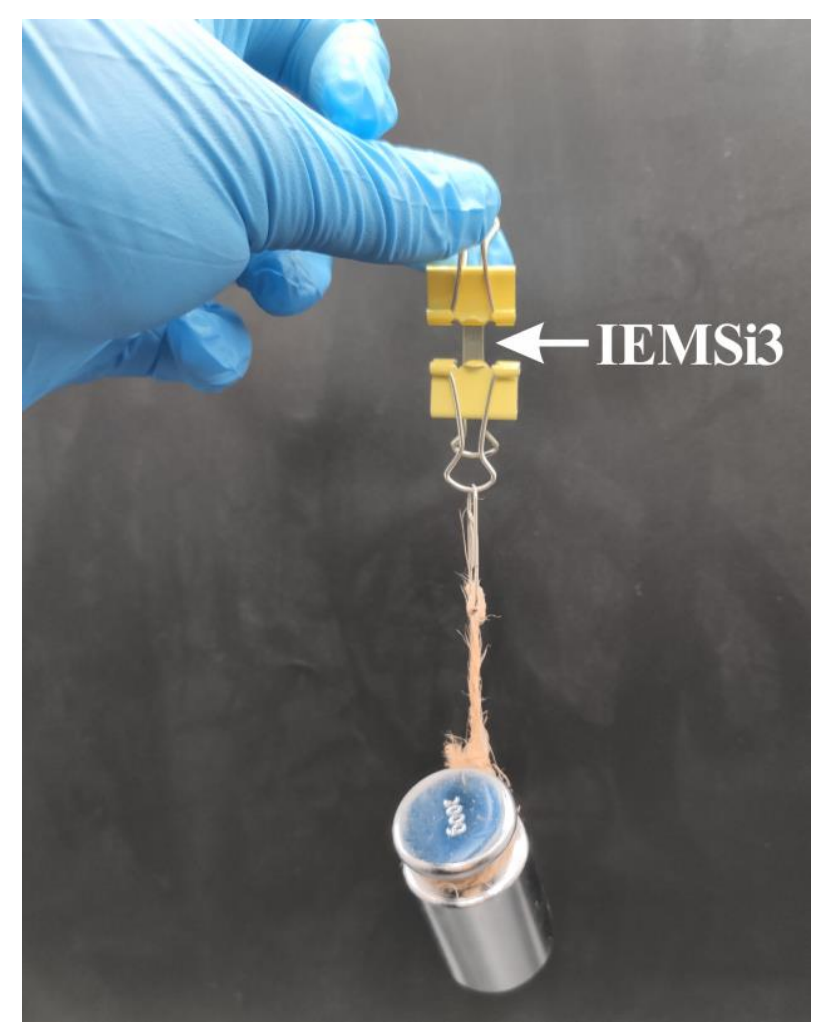

Figure S10. Digital photo of IEMSi3 reconfigured for three times and can still bear a weight of $200 \mathrm{~g}$. 